\title{
FGFR Family Members Protein Expression as Prognostic Markers in Oral Cavity and Oropharyngeal Squamous Cell Carcinoma
}

\author{
Koos Koole ${ }^{1,2}$ - Martijn J. A. M. Clausen ${ }^{3,4}$ - Robert J. J. van Es ${ }^{2}$. \\ Pauline M. W. van Kempen ${ }^{5}$ Lieuwe J. Melchers ${ }^{4} \cdot$ Ron Koole $^{2}$ • \\ Johannes A. Langendijk ${ }^{6}$ - Paul J. van Diest ${ }^{1} \cdot$ Jan L. N. Roodenburg ${ }^{4}$. \\ Ed Schuuring $^{3} \cdot$ Stefan M. Willems ${ }^{1,7}$
}

Published online: 9 June 2016

(C) The Author(s) 2016. This article is published with open access at Springerlink.com

\begin{abstract}
Introduction Fibroblast growth factor receptor family member proteins (FGFR1-4) have been identified as promising novel therapeutic targets and prognostic markers in a wide spectrum of solid tumors. The present study investigates the expression and prognostic value of four FGFR family member proteins in a large multicenter oral cavity squamous cell carcinoma (OCSCC) and oropharyngeal squamous cell carcinoma (OPSCC) cohort.

Methods Protein expression of FGFR1-4 was determined by immunohistochemistry on tissue microarrays containing 951 formalin-fixed paraffin embedded OCSCC and OPSCC tissues from the University Medical Center Utrecht and University Medical Center Groningen. Protein expression was correlated to overall survival using Cox regression models, and bootstrapping was performed as internal validation.

Results FGFR proteins were highly expressed in 39-64\% of OCSCC and 63-79\% of OPSCC. Seventy-three percent (299/412) of OCSCC and $85 \%$ (305/357) of OPSCC highly co-expressed two or more FGFR family member
\end{abstract}

Electronic supplementary material The online version of this article (doi:10.1007/s40291-016-0204-5) contains supplementary material, which is available to authorized users.

Stefan M. Willems

s.m.willems-4@umcutrecht.n

1 Department of Pathology, University Medical Center Utrecht, H4.2.41, Heidelberglaan 100, 3584 CX Utrecht, The Netherlands

2 Department of Head and Neck Surgical Oncology, UMC Utrecht Cancer Center, University Medical Center Utrecht, Heidelberglaan 100, 3584 CX Utrecht, The Netherlands

3 Department of Pathology and Medical Biology, University Medical Center Groningen, University of Groningen, Hanzeplein 1, 9713 GZ Groningen, The Netherlands proteins. FGFR1 protein was more frequently highly expressed in human papillomavirus (HPV)-negative OPSCC than HPV-positive OPSCC (82 vs. $65 \%$; $p=0.008$ ). Furthermore, protein expression of FGFR family members was not related to overall survival in OCSCC or OPSCC $(p>0.05)$.

Conclusion FGFR family members are frequently highly expressed in OCSCC and OPSCC. These FGFR family member proteins are therefore potential targets for novel therapies that are urgently required to improve survival of OCSCC and OPSCC patients.

\section{Key Points}

FGFR family members have been identified as novel therapeutic targets and prognostic markers in multiple types of cancer.

In this study we found high expression of all four FGFR family member proteins in large oral cavity and oropharyngeal squamous cell carcinoma cohorts.

All four FGFR family member proteins may serve as potential therapeutic targets.

4 Department of Oral and Maxillofacial Surgery, University Medical Center Groningen, University of Groningen, Hanzeplein 1, 9713 GZ Groningen, The Netherlands

5 Department of Otorhinolaryngology-Head and Neck Surgery, University Medical Center Utrecht, Heidelberglaan 100, 3584 CX Utrecht, The Netherlands

6 Department of Radiation Oncology, University Medical Center Groningen, University of Groningen, Hanzeplein 1, 9713 GZ Groningen, The Netherlands

7 Department of Molecular Carcinogenesis, Netherlands Cancer Institute, Plesmanlaan 121, 1066 CX Amsterdam, The Netherlands 


\section{Introduction}

Despite improvements in diagnostic tools and treatment regimens over the past three decades, overall survival rates of advanced (stage III-IV) head and neck squamous cell carcinoma (HNSCC) have only reached $65 \%$ [1]. To improve overall survival rates by means of a more individualized treatment, new prognostic markers have been identified for HNSCC and these are currently integrated into HNSCC clinical trials. Prognostic markers include Epstein-Barr virus (EBV) status, human papillomavirus (HPV) status and PET-imaging features [2]. However, despite these advancements, improvement of HNSCC overall survival remains limited. Therefore, novel markers are needed to select HNSCC patients for adjuvant therapy and thereby improve overall survival rates. Generally, HNSCC is treated by surgery and/or chemo-irradiation, which cause severe side-effects in more than $80 \%$ of the advanced HNSCC patients [3]. Unfortunately, until now other treatment options for HNSCC patients are limited to only one single targeted therapy available in the clinic and with variable benefit i.e. cetuximab, targeting epidermal growth factor receptor (EGFR) protein [4]. To expand personalized cancer care for HNSCC, novel targeted therapies are needed [5].

For a wide spectrum of other types of cancer, including lung, breast and colorectal cancer, multiple targeted therapies have been developed and approved. One of these targets for therapies and prognostic markers recently identified, is the fibroblast growth factor receptor (FGFR) protein family [4]. The FGFR family comprises four cell membrane bound tyrosine kinase receptors (FGFR1-4) that activate multiple intracellular signaling pathways. Genomic driver aberrations, such as mutations, amplifications and translocations of FGFRl-4 genes dysregulate FGFR signaling pathways and promote tumor development [6]. Targeting FGFR family members with FGFR-inhibitors has shown promising therapeutic value in clinical trials on breast, colorectal, thyroid and non-small cell lung cancer [7, 8]. Although previous studies have observed prognostic and therapeutic value for FGFR family members, the expression and prognostic value of all four FGFR family member proteins has not been investigated in a cohort of HNSCC so far. To assess their prognostic relevance, we investigated the expression and prognostic value of all four FGFR family member proteins in large cohorts of both oral cavity squamous cell carcinoma (OCSCC) and oropharyngeal squamous cell carcinoma (OPSCC).

\section{Materials and Methods}

\subsection{Patient Cohorts}

Inclusion criteria for the patient cohorts were: patients with a first primary HNSCC of oral cavity or oropharyngeal location who were treated with curative intent at the University Medical Center Utrecht (UMCU) or University Medical Center Groningen (UMCG) between the years 1996 and 2011 (Table 1). Exclusion criteria were: HNSCC of nasopharyngeal, hypopharyngeal, or laryngeal location, a previous history of HNSCC, a synchronous primary tumor, histological abnormalities including dysplastic lesions and inflammation, and the absence of tumor cores on tissue microarray slides (TMA). Clinicopathological data and follow-up data on patient overall survival were retrieved from electronic medical records. Formalin-fixed paraffin-embedded (FFPE) tissues of all tumors were collected from pathology departments. OCSCC tissues included mainly surgical resection specimens and OPSCC tissues included mainly pretreatment biopsy specimens. Human tissues and patient data were used according to "The Code for Proper Secondary Use of Human Tissue" and "The Code of Conduct for the Use of Data in Health Research" as stated by the Federation of Dutch Medical Scientific Societies (Federa FMVV, updated 2011). All slides and diagnoses were reviewed by a dedicated pathologist (SMW). HPV status was determined for tumors using a combination of p16 immunohistochemistry and a PCR-based HPV-genotyping method as described previously [9, 10]. Using the reversed Kaplan-Meier method, median follow-up time of OCSCC patients was 78.5 months and the median follow-up time of OPSCC patients was 57 months.

\subsection{Tissue Microarray Construction}

FFPE tissues were constructed into tissue microarrays using either the TMA Grand Master instrument (3D HISTECH, Budapest, Hungary) in the UMCU or Manual Tissue Arrayer I (Beecher Instruments, Sun Prairie, WI, USA) in the UMCG. Construction of the UMCG-TMA was reported previously $[9,11,12]$. Tumor areas of FFPE tissues were marked by a pathologist (SMW) on the original H\&E slides. Three cores $(0.6 \mathrm{~mm})$ were punched from tumor areas of each FFPE tissue and arrayed into a recipient donor block. Normal placenta, liver, lung, stomach, mammary, appendix, adrenal gland, colon and testis tissues were included into TMAs for quality control of staining 
Table 1 Baseline characteristics of oral cavity squamous cell carcinoma and oropharyngeal squamous cell carcinoma patient cohorts from the University Medical Center Utrecht and University Medical Center Groningen

\begin{tabular}{|c|c|c|c|c|c|}
\hline & UMCU & UMCG & OCSCC & OPSCC & $\begin{array}{l}p \text { (OCSCC vs. } \\
\text { OPSCC) }\end{array}$ \\
\hline Total no. of cases $(n=951)$ & $452(100)$ & $499(100)$ & $512(100)$ & $439(100)$ & \\
\hline \multicolumn{6}{|l|}{ Age } \\
\hline Median (range) & $60(26-88)$ & $60(24-94)$ & $62(24-94)$ & $58(35-89)$ & 0.001 \\
\hline \multicolumn{6}{|l|}{ Gender } \\
\hline Male & $295(65)$ & $312(63)$ & $305(60)$ & $302(69)$ & 0.003 \\
\hline Female & $157(35)$ & $187(37)$ & $207(40)$ & $137(31)$ & \\
\hline \multicolumn{6}{|l|}{ Clinical T-classification } \\
\hline cT1-cT2 & $213(47)$ & $261(52)$ & $310(61)$ & $164(37)$ & $<0.001$ \\
\hline cT3-cT4 & $238(52.5)$ & $227(46)$ & $191(37)$ & $274(62)$ & \\
\hline Missing & $1(0.5)$ & $11(2)$ & $11(2)$ & $1(1)$ & \\
\hline \multicolumn{6}{|l|}{ Clinical N-classification } \\
\hline $\mathrm{cNO}$ & $209(46)$ & $221(44)$ & $334(65)$ & $96(22)$ & $<0.001$ \\
\hline $\mathrm{cN} 1-3$ & $177(39)$ & $278(56)$ & $178(35)$ & $277(63)$ & \\
\hline Missing & $66(15)$ & $0(0)$ & $0(0)$ & $66(15)$ & \\
\hline \multicolumn{6}{|l|}{ Clinical M-classification } \\
\hline cM0 & $407(90)$ & $353(71)$ & $401(78)$ & $359(82)$ & $<0.001$ \\
\hline $\mathrm{cM} 1$ & $11(2.5)$ & $4(1)$ & $0(0)$ & $15(3)$ & \\
\hline Missing & $34(7.5)$ & $142(28)$ & $111(22)$ & $65(15)$ & \\
\hline \multicolumn{6}{|l|}{ Site } \\
\hline Oral cavity & $212(47)$ & $300(60)$ & - & - & - \\
\hline Oropharynx & $240(53)$ & $199(40)$ & - & - & - \\
\hline \multicolumn{6}{|l|}{ HPV status } \\
\hline Positive & $45(10)$ & $53(11)$ & $9(2)$ & $89(20)$ & $<0.001$ \\
\hline Negative & $398(88)$ & $414(83)$ & $475(93)$ & $337(77)$ & \\
\hline Missing & $9(2)$ & $32(6)$ & $28(5)$ & $13(3)$ & \\
\hline \multicolumn{6}{|l|}{ Pathological T-classification } \\
\hline pT1-pT2 & $128(28)$ & $208(42)$ & $294(57)$ & $42(10)$ & 0.036 \\
\hline pT3-pT4 & $112(25)$ & $156(31)$ & $218(43)$ & $50(11)$ & \\
\hline Missing & $212(47)$ & $135(27)$ & $0(0)$ & 347 (79) & \\
\hline \multicolumn{6}{|l|}{ Pathological N-classification } \\
\hline pNO & $104(23)$ & $158(31.5)$ & $233(45.5)$ & $29(6.5)$ & 0.001 \\
\hline $\mathrm{pN} 1-3$ & $129(28.5)$ & $198(39.5)$ & $266(52)$ & $61(14)$ & \\
\hline Missing & $219(48.5)$ & $143(29)$ & $13(2.5)$ & 349 (79.5) & \\
\hline \multicolumn{6}{|l|}{ Pathological stage } \\
\hline I-II & $93(21)$ & $115(23)$ & $158(31)$ & $50(11)$ & $<0.001$ \\
\hline III-IV & $346(76)$ & $384(77)$ & 354 (69) & $376(86)$ & \\
\hline Missing & $13(3)$ & $0(0)$ & $0(0)$ & $13(3)$ & \\
\hline \multicolumn{6}{|l|}{ Primary treatment } \\
\hline Surgery & $273(60)$ & $371(74)$ & $512(100)$ & $132(30)$ & $<0.001$ \\
\hline (Chemo)radiotherapy & $153(34)$ & $103(20.5)$ & $0(0)$ & $256(58.5)$ & \\
\hline Palliative & $26(6)$ & $23(5)$ & $0(0)$ & $49(11)$ & \\
\hline Missing & $0(0)$ & $2(0.5)$ & $0(0)$ & $2(0.5)$ & \\
\hline \multicolumn{6}{|l|}{ Neck dissection } \\
\hline Yes & $233(51)$ & $365(73)$ & $499(97)$ & $99(22.5)$ & $<0.001$ \\
\hline No & $193(43)$ & $109(22)$ & $13(3)$ & $289(66)$ & \\
\hline Palliative & $26(6)$ & $23(4.5)$ & $0(0)$ & $49(11)$ & \\
\hline Missing & $0(0)$ & $2(0.5)$ & $0(0)$ & $2(0.5)$ & \\
\hline
\end{tabular}


Table 1 continued

UMCU

UMCG

OCSCC

OPSCC

$p$ (OCSCC vs.

OPSCC)

Postoperative (chemo)radiotherapy

Yes

104 (23)

268 (54)

283 (55)

89 (20)

0.012

No

169 (37)

$103(20.5)$

229 (45)

43 (10)

Surgery not primary treatment

153 (34)

$103(20.5)$

$0(0)$

$256(58.5)$

Palliative

$26(6)$

$23(4.5)$

$0(0)$

49 (11)

Missing

$0(0)$

$2(0.5)$

$0(0)$

$2(0.5)$

Extra nodal growth

Yes

66 (14.5)

38 (7.5)

93 (18)

$11(2.5)$

0.305

61 (13.5)

55 (11)

Not applicable

104 (23)

158 (31.5)

7 (1.5)

109 (21)

29 (7)

Missing

$221(49)$

248 (50)

233 (46)

392 (89)

Lymphovascular invasion

Yes

$52(11.5)$

44 (9)

$77(15)$

27 (6)

0.023

206 (45.5)

288 (58)

69 (14)

89 (20)

Missing

167 (33)

405 (79)

$323(74)$

Perineural growth

Yes

97 (21.5)

90 (18)

38 (7)

31 (7)

0.171

154 (34)

262 (53)

156 (30)

89 (20)

Missing

201 (44.5)

147 (29)

327 (64)

319 (73)

Bone invasion

Yes

64 (14)

60 (12)

29 (6)

5 (1)

0.001

176 (39)

20 (4)

119 (23)

30 (7)

Missing

212 (47)

419 (84)

191 (37)

404 (92)

Growth pattern

\section{Cohesive}

55 (12)

5 (1)

$202(40)$

$11(2.5)$

49 (10)

33 (7.5)

200 (44)

105 (21)

272 (53)

395 (90)

Missing

197 (44)

389 (78)

191 (37)

$3(0.5)$

50 (10)

43 (10)

161 (32)

337 (66)

393 (89.5)

Missing

219 (48)

$306(61)$

125 (24)

33 (7.5)

0.007

Well/moderate

189 (42)

260 (52)

$416(81)$

$16(3.5)$

50 (11)

51 (10)

85 (17)

390 (89)

Missing

213 (47)

188 (38)

11 (2)

$27(0-168)$

$<0.001$

Median (range)

43.5 (0-185)

$33(0-167)$

$43(0-185)$

202 (46)

0.026

\begin{tabular}{lccll} 
Alive & $185(41)$ & $296(59)$ & $278(54)$ & $202(46)$ \\
Dead & $241(53)$ & $175(35)$ & $230(45)$ & $186(42.5)$ \\
Palliative & $26(6)$ & $23(5)$ & $0(0)$ & $49(11)$ \\
Missing & $0(0)$ & $5(1)$ & $4(1)$ & $2(0.5)$ \\
\hline
\end{tabular}

The cohorts comprised oral cavity and oropharyngeal squamous cell carcinoma patients, who were treated in these hospitals between the year 1996 and 2011. Clinicopathological characteristics of all patients were retrieved from electronic medical records and formalin fixed paraffinembedded tissues of all tumors were collected. The human papillomavirus type (HPV) status was determined using P16 immunohistochemistry and a PCR-based HPV-genotyping method

$H P V$ human papillomavirus, $O C S C C$ oral cavity squamous cell carcinoma, $O P S C C$ oropharyngeal squamous cell carcinoma, UMCG University Medical Center Groningen, UMCU University Medical Center Utrecht 
[13]. Normal healthy tonsillar and oral mucosa tissues were included in all TMAs. These tissues were collected from healthy individuals who have no history of HNSCC.

\subsection{Immunohistochemistry}

To determine FGFR family member protein expression, immunohistochemistry was performed on TMA slides with a BenchMark ULTRA automated staining instrument (Ventana Medical Systems, Tucson, AZ, USA). Slides were mechanically deparaffinized, pretreated with EDTA and rinsed with reaction buffer. Next, sets of slides were incubated with $150 \mu \mathrm{L}$ anti-FGFR1 antibody (ab10646, 1:2000 dilution) (Abcam, Cambridge, UK), anti-FGFR2 antibody (ab10648, 1:1000 dilution) (Abcam), anti-FGFR3 antibody (sc-13121, 1:25 dilution) (Santa Cruz Biotechnology, Dallas, TX, USA) or anti-FGFR4 antibody (PAB3044, 1:200 dilution) (Abnova, Taipei City, Taiwan) for 32 min. Prior to staining TMA slides, staining methods were optimized by testing antibodies for pretreatments EDTA, citrate, pepsine, or no pretreatment with dilutions ranging from $1: 10$ to $1: 2000$. Antibody specificity to the corresponding FGFR protein was verified by testing them on the following positive and negative controls; placenta and stomach for FGFR1, lung and pancreas for FGFR2, liver and stomach for FGFR3, and stomach and lung for FGFR4 [13]. After rinsing with reaction buffer, slides were incubated with Optiview HQ Universal Linker and Optiview HRP multimer (Ventana Medical Systems) for eight min. Finally, diaminobenzidine was applied and slides were counterstained with hematoxylin.

To semi-quantify FGFR1-4 protein expression, the percentage of positively stained tumor cells $(0-100 \%)$ and staining intensity (negative/normal healthy tissue 0 , moderate +1 , strong +2 ) were scored in OCSCC and OPSCC. FGFR1-4 staining in OCSCC and OPSCC was compared to staining in normal healthy tonsillar and oral mucosa tissues. Staining in these normal tissues was used as a reference. All TMA cores were scored by a dedicated head and neck pathologist (SMW) and head and neck researchers (KK and MJAMC) together. All three observers were blinded to the outcome of patients. Discordant cases were discussed until consensus was reached. For FGFR3, only the staining intensity was scored as the percentage of stain-positive tumor cells was always $100 \%$ and thus not discriminative. For each tumor, mean percentages and maximum stain intensities were computed from available TMA cores. Tumors with only one or two TMA cores available were included in the analysis. Continuous percentage scores were dichotomized by cutoff values, which were optimized for predicting patient overall survival using log-likelihood values. Optimizing cutoff values on patient outcome using log-likelihood values has been described as the appropriate method in previous studies [14]. Cutoff values for both OCSCC and OPSCC were $10 \%$ for FGFR $1,15 \%$ for FGFR2 and $33 \%$ for FGFR4. Other cutoff values and the staining intensity for FGFR1, FGFR2 and FGFR4 were much less optimal in predicting overall survival (data not shown) and were not further used in this study. For FGFR3, +1 intensity was selected as a cutoff value, with 0 defined as low/negative expression and +1 and above defined as high expression. Co-expression of FGFR family member proteins was only evaluated in tumors for which data on all four FGFRs were available as for some tumors TMA cores were missing. Co-expression was defined as concurrent high expression of two or more FGFR family member proteins in a single tumor.

\subsection{Statistical Analysis}

Data were analyzed using IBM SPSS Statistics version 22 software (IBM, Amonk, NY, USA). Baseline characteristics and protein expression were compared between OCSCC and OPSCC using Pearson's chi square test for dichotomous variables and t-test for continuous variables.

Patients who were treated with curative intent were included and patients who were treated with palliative intent were excluded from overall survival analysis. Univariate Kaplan-Meier overall survival curves were plotted and compared with a log-rank test to analyze the relationships between protein expression of FGFR family members and overall survival. Significant relationships for FGFR1 expression and FGFR1-4 co-expression were further analyzed by multivariate Cox regression. Covariates were detected by analyzing relationships between variables and overall survival, and confounders were detected by analyzing variables in relation to FGFR expression and overall survival. Covariates and confounders included in both multivariate models were: clinical T-classification, clinical N-classification, pathological T-classification, pathological N-classification, extra nodal growth, lymphovascular invasion, perineural growth, growth pattern and pathological stage. Postoperative (chemo)radiotherapy was identified as an effect modifier for FGFR1-4 protein co-expression, and corrected for in the model. Internal validation of the (bio)marker-based risk prediction model was performed by bootstrapping based on 5000 samples. Splitting the study cohort in a development and validation cohort was not the preferred validation method, because it is statistically inefficient to only use a subset of all data to produce the prediction model and it is accompanied by replication instability, as described by Moons et al. [15]. Two-sided $p$ values below 0.05 were considered significant throughout all statistical computations. The $p$ values were corrected for multiple-comparison by calculating Bonferroni-corrected $p$ values as appropriate. Finally, Python 
A

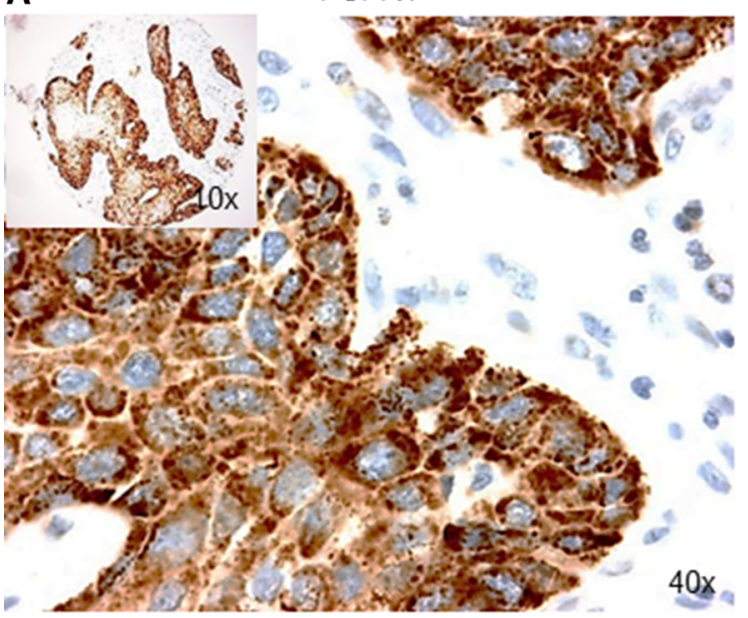

C

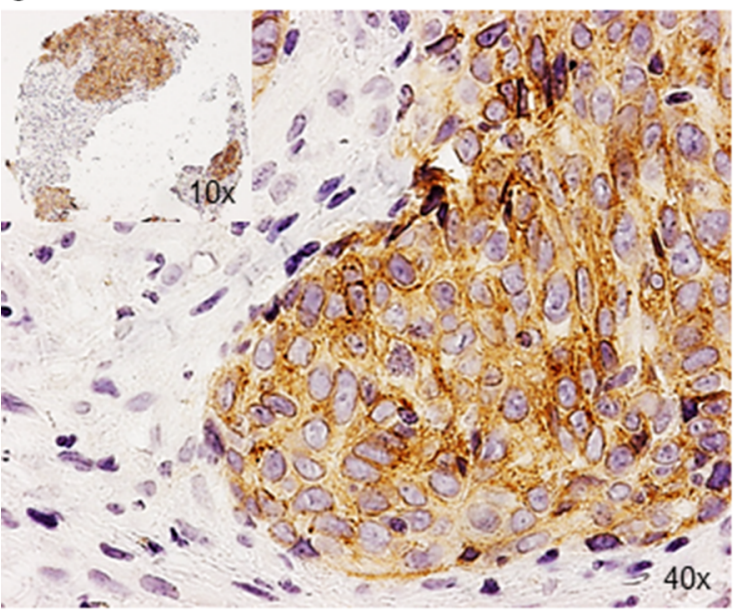

E

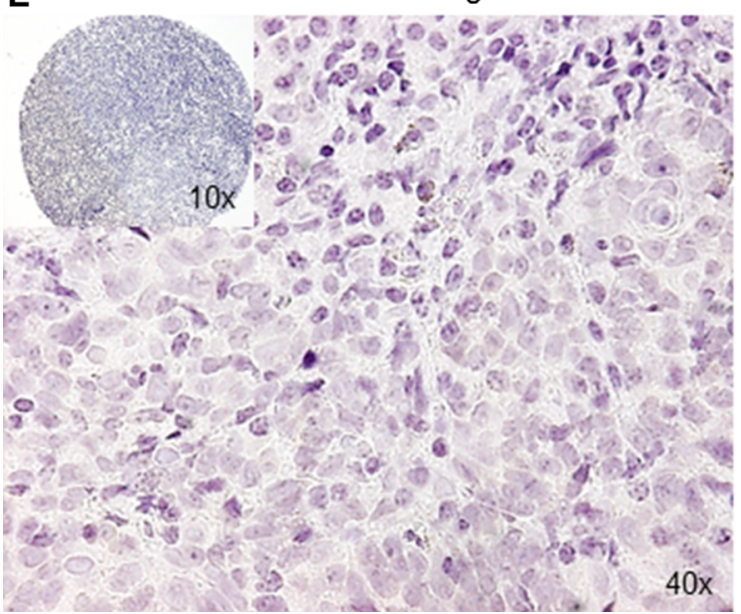

Fig. 1 Representative microscopic images of immunohistochemical staining for FGFR family member proteins in oral cavity squamous cell carcinoma and normal healthy oral mucosa tissue microarray cores ( $\times 10$ and $\times 40$ magnification). a-d Strong immunohistochemical staining indicating high FGFR1, FGFR2, FGFR3 and FGFR4 protein expression in oral cavity squamous cell carcinoma. e No immunohistochemical staining indicating absence of FGFR1-4
B

FGFR2

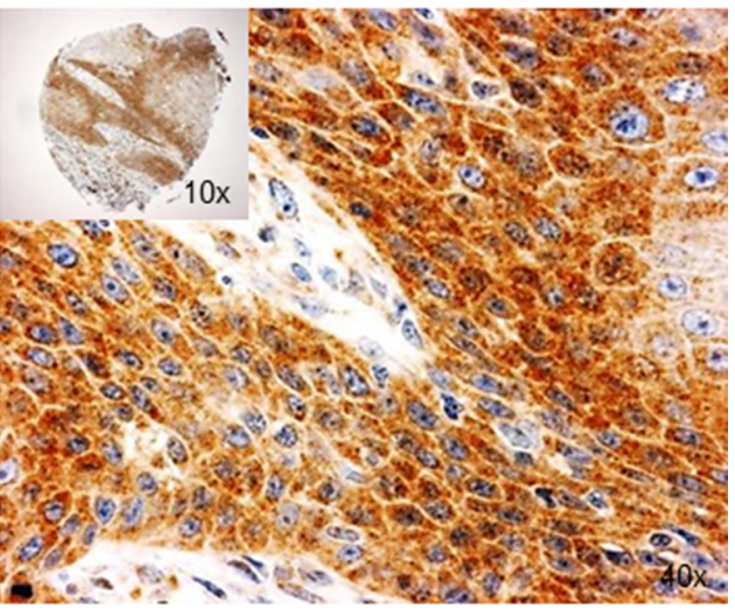

D

FGFR4

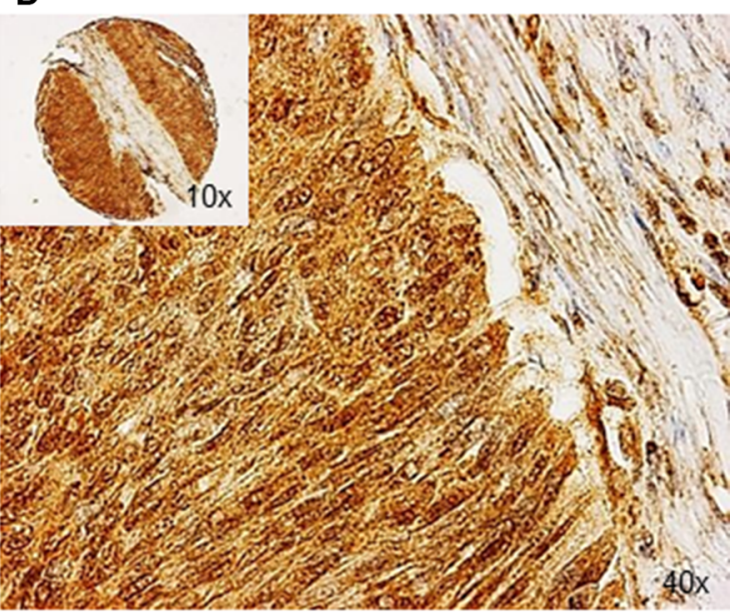

F Normal healthy oral mucosa tissue

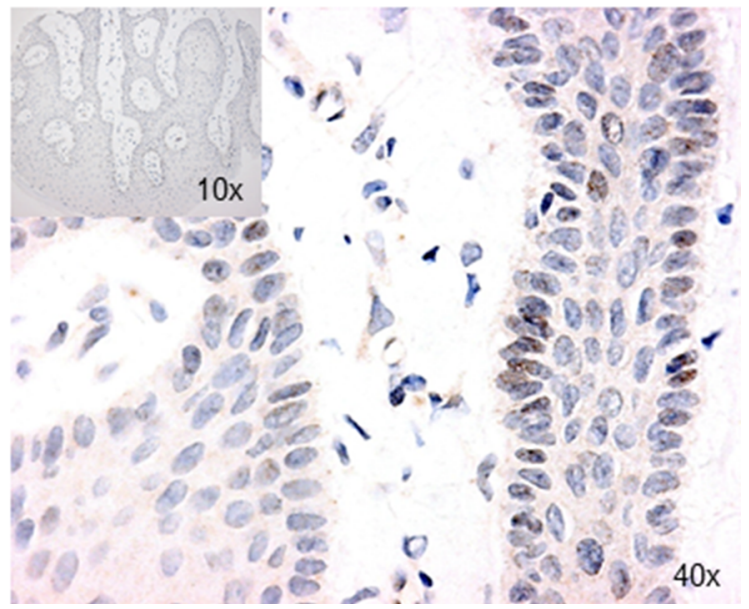

protein expression in oral cavity squamous cell carcinoma. f Faint immunohistochemical staining indicating low expression of FGFR1-4 protein in normal healthy oral mucosa tissue. Protein expression was determined immunohistochemically using antiFGFR1-4 antibodies in a cohort of oral cavity squamous cell carcinoma $(n=512)$ and oropharyngeal squamous cell carcinoma $(n=439) . F G F R$ fibroblast growth factor receptor 
version 2.7 was used to analyze co-high expression data and $\mathrm{R}$ package VennDiagram version 3.1.2 was used to plot Venn diagrams.

\section{Results}

\subsection{Clinicopathological Characteristics}

The UMC Utrecht cohort included 212 OCSCC and 240 OPSCC patients and the UMC Groningen cohort included 300 OCSCC and 199 OPSCC patients. Altogether, the total cohort comprised 512 OCSCC patients and 439 OPSCC patients. Twenty-one percent $(89 / 426)$ of OPSCC were HPV-positive, $79 \%$ (337/426) HPV-negative, and $3 \%$ (13/ 439) were not tested for HPV. Thirty-one percent (158/512) of OCSCC were early (stage I-II) tumors and $69 \%$ (354/ 512 ) advanced (stage III-IV) tumors. Twelve percent (50/ 426 ) of OPSCC were early (stage I-II) tumors and $88 \%$ (376/426) advanced (stage III-IV) tumors, and pathological stage was missing in $3 \%$ (13/439) of OPSCC. One hundred percent $(512 / 512)$ of OCSCC were treated with surgery, $97 \%(499 / 512)$ received a neck dissection and $55 \%$ (283/ 512) received postoperative (chemo)radiotherapy. Thirty percent $(132 / 437)$ of OPSCC were treated with surgery, $59 \%$ (256/437) with (chemo)radiotherapy, $11 \%$ (49/437) were treated with palliative intent, and the type of primary treatment was missing in $0.5 \%$ (2/439). Twenty-three percent (99/437) of OPSCC received a neck dissection and $20 \%$ (89/437) received postoperative (chemo)radiotherapy. Table 1 summarizes the clinicopathological and follow-up data of the OCSCC and OPSCC cohorts.

\subsection{FGFR1-4 Proteins are Frequently High Expressed and Co-Expressed in Oral Cavity and Oropharyngeal Squamous Cell Carcinoma}

Representative images of FGFR proteins high expressed and low expressed in OCSCC and OPSCC are shown in Fig. 1a-e. Mainly cytoplasmic staining was observed, as reported in gastric cancer [16]. Faint FGFR1-4 staining of 0 intensity was observed in all normal healthy oral mucosa and tonsillar tissues, indicating low expression found in these tissues (Fig. 1f). Strong staining for FGFR family member proteins was noted in corresponding positive controls and absence of staining was noted in corresponding negative controls.

FGFR proteins were highly expressed in 39-64\% of OCSCC (FGFR4 and FGFR1 respectively) and 63-79\% of OPSCC (FGFR3 and FGFR1 respectively) (Fig. 2 and Supplementary Table S1). High protein expression was more common in OPSCC than OCSCC for all four FGFR family members (FGFR1-4: $p=0.008$ ). Within the OPSCC

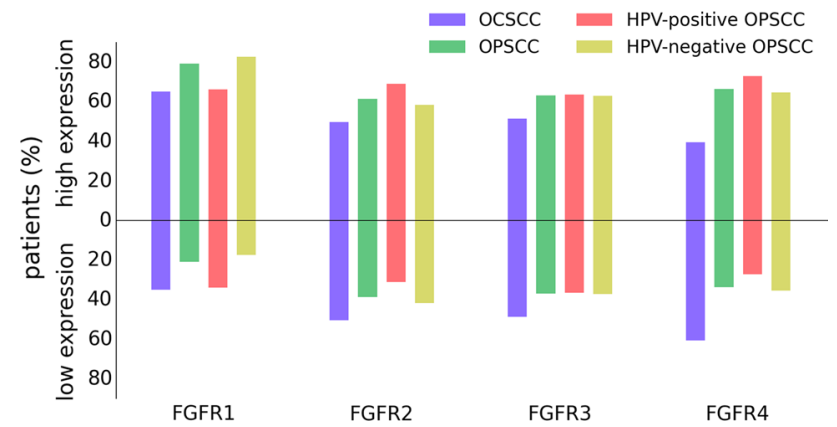

Fig. 2 Expression of FGFR1-4 protein sorted by head and neck cancer site and HPV status. FGFR1-4 protein expression was determined immunohistochemically in a cohort of oral cavity squamous cell carcinoma $(n=512)$ and oropharyngeal squamous cell carcinoma $(n=439)$ from the University Medical Center Utrecht and University Medical Center Groningen. FGFR1-4 proteins were high expressed in 39-64\% of oral cavity squamous cell carcinoma and $63-79 \%$ of oropharyngeal squamous cell carcinoma. High protein expression was more common in oropharyngeal squamous cell carcinoma than oral cavity squamous cell carcinoma for all four FGFR family members $(p=0.008)$. FGFR1 protein was high expressed much more frequently in HPV-negative than in HPVpositive oropharyngeal squamous cell carcinoma (82 vs. $65 \%$; $p=0.008)$. FGFR fibroblast growth factor receptor, $H P V$ human papillomavirus, OPSCC oropharyngeal squamous cell carcinoma, OCSCC oral cavity squamous cell carcinoma

population, FGFR1 protein was highly expressed more frequently in HPV-negative than in HPV-positive OPSCC (82 vs. $65 \% ; p=0.008)$. Expression of FGFR proteins was missing for a subgroup of OCSCC and OPSCC because all three TMA cores were missing (Supplementary Table S1).

Furthermore, two or more FGFR proteins were highly co-expressed in $73 \%$ (299/412) of OCSCC and $85 \%$ (305/ 357) of OPSCC (Fig. 3). FGFR $1 / 2$ were most frequently highly co-expressed in OCSCC $(13 \% ; 55 / 412)$ and FGFR $1-4$ in OPSCC $(28 \% ; 101 / 357)$. Within the OPSCC population, FGFR $2 / 3$ were more frequently highly co-expressed in HPV-positive compared to HPV-negative OPSCC (5\% (4/73) versus $0.4 \%$ (1/275); OR 15.81; $95 \%$ CI $1.75-143 ; p=0.014)$ but lost significance after correcting for multiple testing. Expression of one or more FGFR proteins was missing in $20 \%$ (100/512) of OCSCC and $19 \%(82 / 439)$ of OPSCC because all three TMA cores were missing for these tumors.

\subsection{High FGFR Expression is Not Related to Poor Overall Survival in Oral Cavity and Oropharyngeal Squamous Cell Carcinoma}

High FGFR1 expression $(p=0.018)$ and high FGFR1-4 co-expression (0.030) was related to poor overall survival in OCSCC in univariate analysis, but lost significance in multivariate models (FGFR1: OR 1.46; $95 \%$ CI $0.91-2.34 ; p=0.690$, FGFR1-4: OR: $2.44 ; 95 \%$ CI 1.29-5.50; $p=0.060$ ) (Table 2; Fig. 4). All other FGFR 

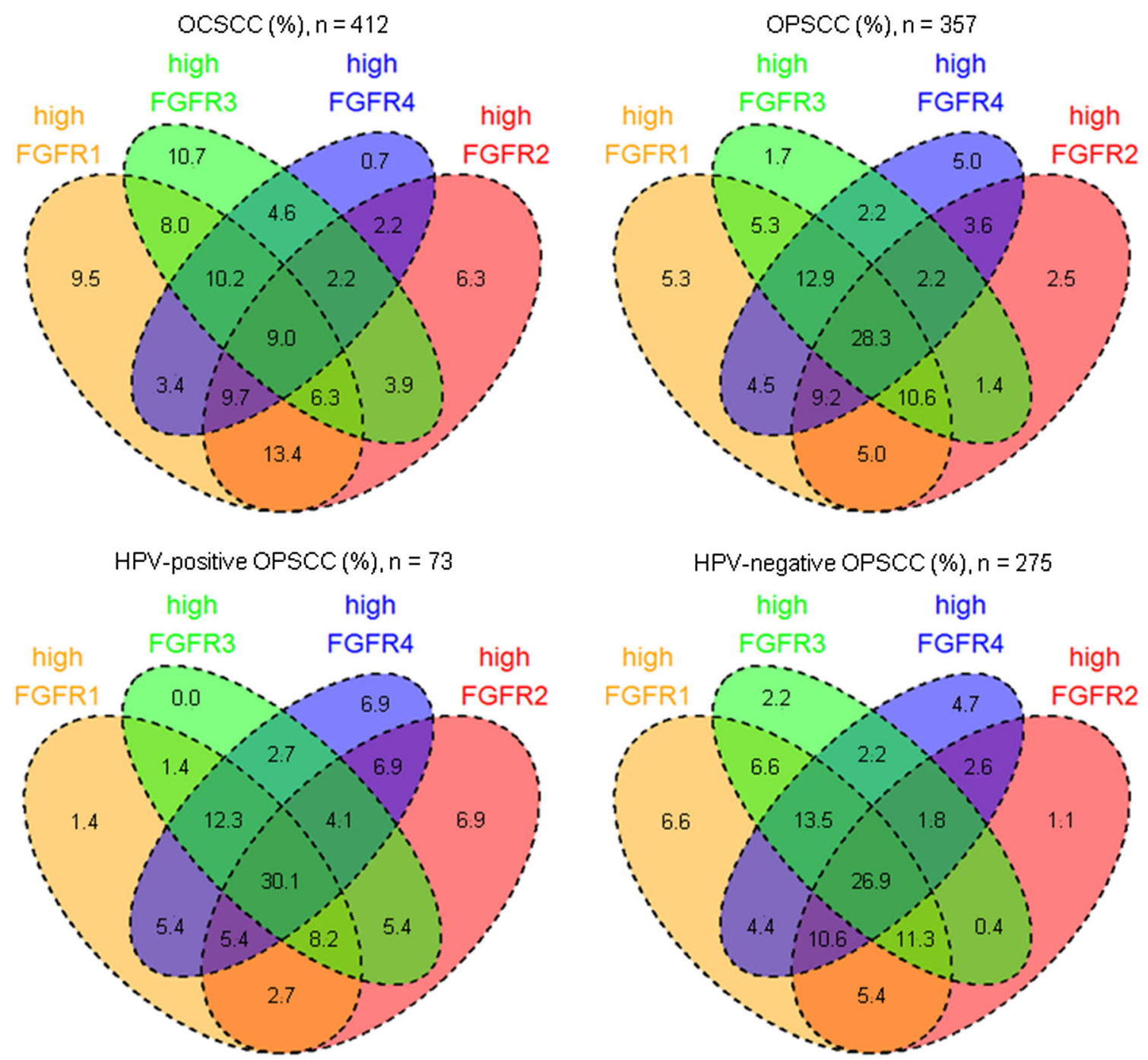

Fig. 3 Venn diagrams of high FGFR1-4 protein co-expression sorted by head and neck cancer site and HPV status. FGFR1-4 protein expression was determined immunohistochemically in a cohort of oral cavity squamous cell carcinoma $(n=512)$ and oropharyngeal squamous cell carcinoma $(n=439)$. FGFR1 protein was highly expressed more frequently in HPV-negative than in HPV-positive oropharyngeal squamous cell carcinoma (82 vs. $65 \% ; p=0.008$ ). Furthermore, FGFR $2 / 3$ were more frequently highly co-expressed in HPV-positive compared to HPV-negative oropharyngeal squamous

cell carcinoma $[5 \%(4 / 73)$ vs. $0.4 \%(1 / 275)$; OR $15.81 ; 95 \% \mathrm{CI}$ $1.75-143 ; p=0.014]$, but lost significance after correcting for multiple testing. Co-expression was only assessed in samples in which expression data for all four FGFR family members was available. Co-expression was defined as concurrent high expression of two or more FGFR family member proteins in a single tumor. FGFR fibroblast growth factor receptor, $H P V$ human papillomavirus, OPSCC oropharyngeal squamous cell carcinoma, OCSCC oral cavity squamous cell carcinoma

family members were not related to overall survival in OCSCC, OPSCC, HPV-positive OPSCC and HPV-negative OPSCC (Table 2; Fig. 4, Supplementary Fig. 1). Expression of FGFR family member proteins was not related to pathological stage $(p>0.05)$.

\section{Discussion}

We identified frequent high expression of all four FGFR family member proteins in large cohorts of OCSCC and OPSCC, with significant higher expression rates in

OPSCC. Previous studies have reported high FGFR1 and FGFR4 expression in HNSCC but at much lower rates of $12 \%$ for FGFR 1 by Freier et al. and $16 \%$ for FGFR 4 by Streit et al., compared to 64 and $39 \%$ in this study [17-20]. Disagreements in high expression rates between studies could be explained by the use of different cutoff values, antibodies and patient cohorts.

In this study, no prognostic value was observed for protein expression of FGFR family members in OCSCC and OPSCC. This is in contrast to the prognostic value reported for FGFR4 in HNSCC and other FGFR family members in multiple types of cancer including gastric, 
Table 2 Multivariate overall survival Cox regression analysis for protein expression of FGFR family members in oral cavity and oropharyngeal squamous cell carcinoma after internal validation by bootstrapping and Bonferroni correction

\begin{tabular}{llllllc}
\hline Protein (co-)expression & HR & $95 \%$ CI & $p$ & HR & $95 \%$ CI & $p$ \\
\hline & \multicolumn{2}{l}{ OCSCC $(n=508)$} & & \multicolumn{2}{l}{ OPSCC $(n=388)$} & \\
FGFR1 & 1.46 & $0.91-2.34$ & 0.690 & 1.42 & $0.93-2.16$ & 0.648 \\
FGFR2 & 2.03 & $0.64-5.23$ & 0.408 & 1.02 & $0.75-1.40$ & 1.000 \\
FGFR3 & 1.22 & $0.92-1.63$ & 0.966 & 1.09 & $0.79-1.50$ & 1.000 \\
FGFR4 & 1.20 & $0.75-1.91$ & 1.000 & 1.13 & $0.81-1.57$ & 1.000 \\
FGFR1-2 & 1.04 & $0.67-1.61$ & 1.000 & 0.71 & $0.31-1.61$ & 1.000 \\
FGFR1-4 & 2.44 & $1.29-5.50$ & 0.060 & 1.05 & $0.74-1.49$ & 1.000 \\
& HPV-positive OPSCC $(n=80)$ & HPV-negative OPSCC $(n=295)$ \\
FGFR1 & 1.85 & $0.52-6.56$ & 1.000 & 1.21 & $0.75-1.93$ & 1.000 \\
FGFR2 & 1.23 & $0.38-3.91$ & 1.000 & 1.06 & $0.76-1.47$ & 1.000 \\
FGFR3 & 1.00 & $0.34-2.94$ & 1.000 & 1.16 & $0.82-1.63$ & 1.000 \\
FGFR4 & 0.72 & $0.23-2.27$ & 1.000 & 0.83 & $0.38-1.82$ & 1.000 \\
FGFR1-2 & 35.44 & $4.89-256.84$ & 0.006 & 0.30 & $0.09-0.93$ & $0.228^{\mathrm{a}}$ \\
FGFR1-4 & 1.03 & $0.34-3.06$ & 1.000 & 2.20 & $0.49-9.85$ & 1.000 \\
\hline Association
\end{tabular}

Associations between protein expression of FGFR family members and overall survival were analyzed using Cox regression. Significant relationships were further analyzed by multivariate Cox regression models. Covariates and confounders were identified and included in multivariate models. For the relationship between FGFR1-4 protein co-expression and overall survival, postoperative chemotherapy or chemoradiotherapy was identified as an effect modifiers and corrected for in the model. Internal validation of (bio)marker-based risk prediction models was performed by bootstrapping based on 5000 samples Throughout these statistical computations, two-sided $p$ values below 0.05 were considered significant. Cutoff values for protein expression of FGFR family members were optimized on predicting patient outcome. Cutoff values were $10 \%$ for FGFR1, $15 \%$ for FGFR2, +1 intensity for FGFR3 and $33 \%$ for FGFR4. Overall survival status was missing for four oral cavity and two oropharyngeal squamous cell carcinoma patients and HPV status was missing for 13 oropharyngeal squamous cell carcinoma patients. Forty-nine oropharyngeal squamous cell carcinoma patients were excluded from survival analysis because they were treated with palliative intent

$95 \%$ CI $95 \%$ confidence interval, FGFR fibroblast growth factor receptor, HPV human papillomavirus, $H R$ hazard ratio, OPSCC oropharyngeal squamous cell carcinoma, OCSCC oral cavity squamous cell carcinoma

${ }^{a}$ Too few cases were available (FGFR1-2 co-high expression: HPV-positive OPSCC: 2 cases and HPVnegative OPSCC: 3 cases) colorectal, bladder and esophageal cancer [16, 21-25]. In this study, cutoff values for FGFR expression were selected on the value best predicting overall survival outcome. Cutoff values were computed from the same dataset as the results on overall survival were retrieved, by which the observed effects are overestimated. Overestimation of the effects has been corrected for by internal validation, but further external validation on a different cohort is necessary. Secondly, our findings on protein expression of FGFR family members should be confirmed in future studies using other laboratory methods than immunohistochemistry with antibodies.

Interestingly, in our study FGFR1-4 proteins are frequently highly co-expressed in HNSCC, which is in line with similar findings in gastric cancer [25]. The high frequency of FGFR co-expression suggests therapeutic value for FGFR-inhibitors in OCSCC and OPSCC that target multiple FGFR family member proteins. Currently, the most impressive anti-tumor activity has been reported for non-selective tyrosine kinase inhibitors (TKI) but they cause more adverse effects due to vascular endothelial growth factor (VEGF) inhibition. Conversely, selective FGFR-inhibitors show less anti-tumor activity but fewer adverse effects [6]. Selective FGFR-inhibitors are widely available for FGFR1-3 and only recently available for FGFR4 [26, 27]. Therapeutic value has already been observed for the FGFR1-inhibitor PD173074 in HNSCC cell lines and a clinical trial on HNSCC patients with the FGFR-inhibitor ponatinib has been completed [28] (ClinicalTrials.gov Identifier: NCT01761747).

Remarkably, FGFR1 protein was more frequently highly expressed in HPV-negative than HPV-positive OPSCC. This may reflect the FGFR1-driven tumor biology of HPVnegative OPSCC and could identify a specific subgroup sensitive to FGFR1-inhibitor therapy. Several studies have reported genomic aberrations of FGFR1/2/3 unique for HPV-positive or HPV-negative HNSCC and a direct relation between HPV oncogenes E6/7 and the beta-variants of FGFR1-4 expression in five cancer cell lines [29-31]. In these studies, they observed that FGFRI amplification was 

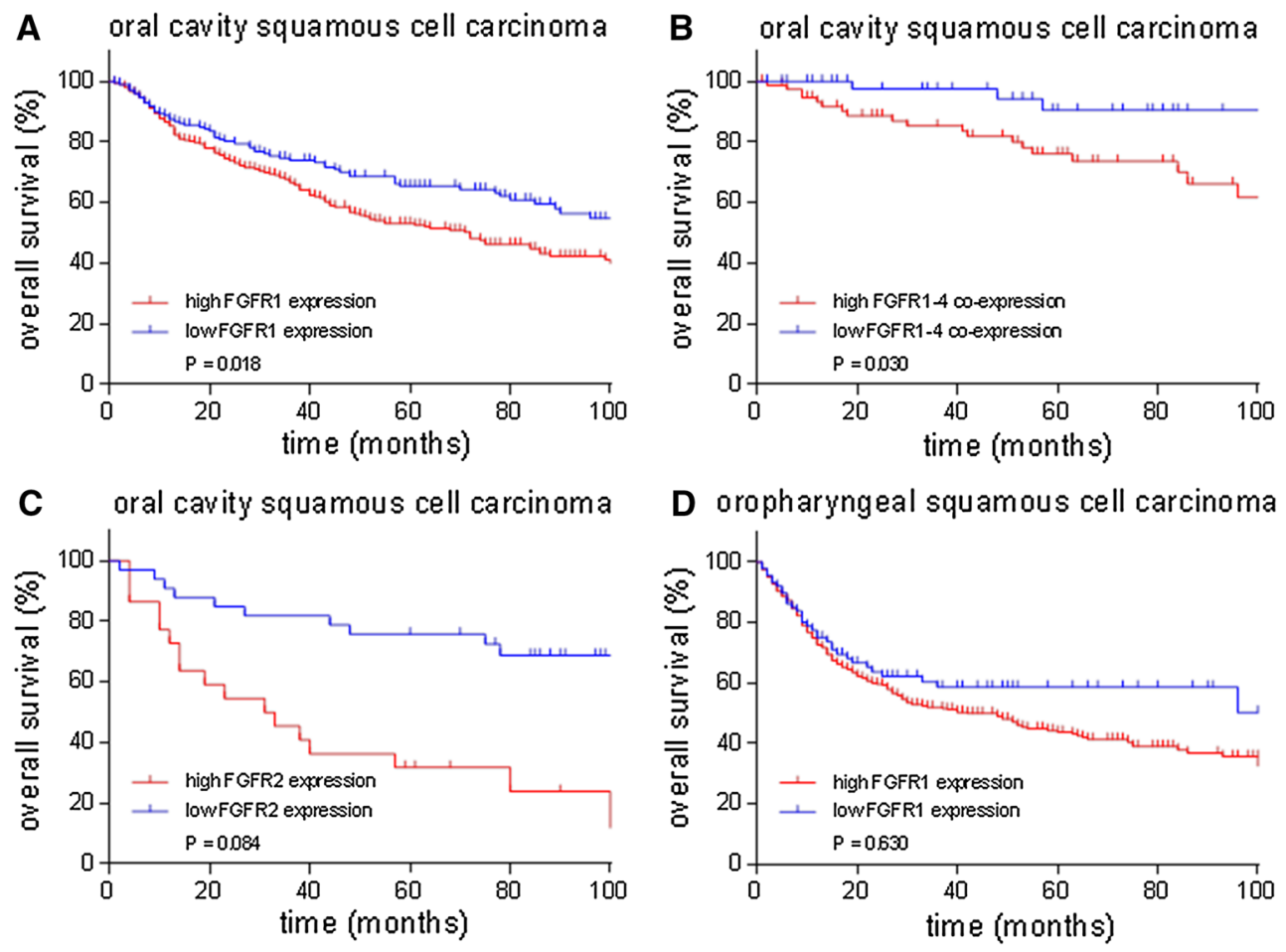

E oropharyngeal squamous cell carcinoma

F oropharyngeal squamous cell carcinoma
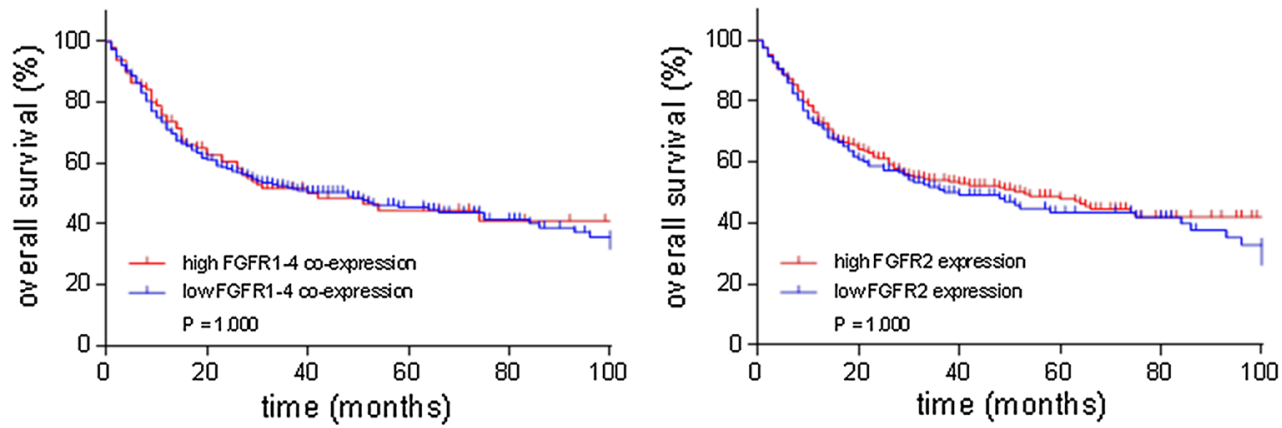

Fig. 4 Kaplan-Meier overall survival curves for FGFR1 and FGFR2 protein expression and FGFR1-4 protein co-expression in oral cavity and oropharyngeal squamous cell carcinoma. a High FGFR1 $(p=0.018)$ protein expression and b high FGFR1-4 $(p=0.030)$ co-expression were related to poor overall survival in oral cavity squamous cell carcinoma in univariate analysis, but lost significance in multivariate analysis (FGFR1: HR 1.46; $95 \%$ CI 0.91-2.34; $p=0.690$, high expression: $151 / 305$ died, low expression: $64 / 172$ died, FGFR1-4: HR 2.44; $95 \%$ CI 1.29-5.50; $p=0.060$, high expression: 25/37 died, low expression: 165/399 died). c-f High

FGFR2 ( $\mathrm{P}=0.084)$ protein expression was not related to overall survival in oral cavity squamous cell carcinoma (high expression: 118/238 died, low expression: 94/239 died). High FGFR1 $(p=0.630)$ expression, high FGFR1-4 $(p=1.000)$ co-expression and high FGFR2 $(p=1.000)$ expression were not related to overall survival in oropharyngeal squamous cell carcinoma (FGFR1: high expression: 148/287 died, low expression: 25/76 died, FGFR1-4: high expression: 45/88 died, low expression: 107/224 died and FGFR2: high expression: 107/225 died, low expression: 61/127 died)

much more common in HPV-negative HNSCC, while $F G F R 2$ and FGFR 3 aberrations were much more common in HPV-positive HNSCC [29, 30]. Also in cervical squamous cell carcinoma, all tumors harboring FGFR3 mutations were HPV-positive [32]. These genomic findings reflect the FGFR1-driven tumor biology of HPV-negative and FGFR2/3-driven tumor biology of HPV-positive HNSCC. The therapeutic implications regarding sensitivity to targeted therapies may correspond accordingly. As such,

our findings on protein level are comparable to genomic findings in literature. So far, clinical trials focus on FGFR genomic aberrations to predict response of HNSCC to FGFR-inhibitors (ClinicalTrials.gov Identifier: NCT01761747). But these predictive markers should be revised, as FGFR1 mRNA and protein expression, rather than FGFRl gene copy-number status, predicted response of HNSCC cell lines to FGFR-inhibitor BGJ398 in a recent report [33]. 
In conclusion, FGFR family members are frequently overexpressed in HNSCC, especially in OPSCC, and expression varies in subpopulations of OCSCC and OPSCC. FGFR family member proteins are therefore potential targets for novel targeted therapies that are urgently needed to improve survival of OCSCC and OPSCC patients.

\section{Compliance with Ethical Standards}

Conflict of interest K. Koole, M.J.A.M. Clausen, R.J.J. van Es, P.M.W. van Kempen, L.J. Melchers, R. Koole, J.A. Langendijk, P.J. van Diest, J.L.N. Roodenburg, E. Schuuring and S.M. Willems had no conflict of interest to declare.

Funding SMW is funded by the Dutch Cancer Society (clinical fellowship: 2011-4964).

Ethical approval and informed consent The Federa code for proper secondary use of human tissue and the medical ethical code of the University Medical Center Utrecht have been obeyed in conduct of the present study.

Open Access This article is distributed under the terms of the Creative Commons Attribution-NonCommercial 4.0 International License (http://creativecommons.org/licenses/by-nc/4.0/), which permits any noncommercial use, distribution, and reproduction in any medium, provided you give appropriate credit to the original author(s) and the source, provide a link to the Creative Commons license, and indicate if changes were made.

\section{References}

1. Pulte D, Brenner H. Changes in survival in head and neck cancers in the late 20th and early 21st century: a period analysis. Oncologist. 2010;15:994-1001.

2. Kang H, Kiess A, Chung $\mathrm{CH}$. Emerging biomarkers in head and neck cancer in the era of genomics. Nat Rev Clin Oncol. 2015;12:11-26.

3. Conley BA. Treatment of advanced head and neck cancer: what lessons have we learned? J Clin Oncol. 2006;24:1023-5.

4. Huang M, Shen A, Ding J, Heng M. Molecularly targeted cancer therapy: some lessons from the past decade. Trends Pharmacol Sci. 2014;35:41-50.

5. Razzouk S. Translational genomics and head and neck cancer: toward precision medicine. Clin Genet. 2014;86:412-21.

6. Dieci MV, Arnedos M, Andre F, Soria JC. Fibroblast growth factor receptor inhibitors as a cancer treatment: from a biologic rationale to medical perspectives. Cancer Discov. 2013;3:264-79.

7. Andre F, Bachelot T, Campone M, Dalenc F, Perez-Garcia JM, Hurvitz SA, Turner N, Rugo H, Smith JW, Deudon S, Shi M, Zhang Y, et al. Targeting FGFR with dovitinib (TKI258): preclinical and clinical data in breast cancer. Clin Cancer Res. 2013;19:3693-702.

8. Soria JC, DeBraud F, Bahleda R, Adamo B, Andre F, Dienstmann R, Delmonte A, Cereda R, Isaacson J, Litten J, Allen A, Dubois F, et al. Phase I/IIa study evaluating the safety, efficacy, pharmacokinetics, and pharmacodynamics of lucitanib in advanced solid tumors. Ann Oncol. 2014;25:2244-51.

9. Melchers LJ, Mastik MF, Samaniego Cameron B, van Dijk BA, de Bock GH, van der Laan BF, van der Vegt B, Speel EJ, Roodenburg JL, Witjes MJ, Schuuring E. Detection of HPV- associated oropharyngeal tumours in a 16-year cohort: more than meets the eye. Br J Cancer. 2015;112:1349-57.

10. Van Kempen PM, van Bockel L, Braunius WW, Moelans CB, van Olst M, de Jong R, Stegeman I, van Diest PJ, Grolman W, Willems SM. HPV-positive oropharyngeal squamous cell carcinoma is associated with TIMP3 and CADM1 promoter hypermethylation. Cancer Med. 2014;3:1185-96.

11. Melchers LJ, Clausen MJ, Mastik MF, Slagter-Menkema L, Langendijk JA, van der Laan BF, van der Wal JE, van der Vegt B, Roodenburg JL, Schuuring E. Head and neck squamous cell carcinomas do not express EGFRvIII. Int J Radiat Oncol Biol Phys. 2014;90:454-62.

12. Pattje WJ, Schuuring E, Mastik MF, Slagter-Menkema L, Schrijvers ML, Alessi S, van der Laan BF, Roodenburg JL, Langendijk JA, van der Wal JE. The phosphatase and tensin homologue deleted on chromosome 10 mediates radiosensitivity in head and neck cancer. Br J Cancer. 2010;102:1778-85.

13. Hughes SE. Differential expression of the fibroblast growth factor receptor (FGFR) multigene family in normal human adult tissues. J Histochem Cytochem. 1997;45:1005-19.

14. Williams BA, Mandrekar JN, Mandrekar SJ, Cha SS, Furth AF. Finding optimal cutpoints for continuous covariates with binary and time-to-event outcomes. Rochester: Mayo Foundation; 2006.

15. Moons KG, Kengne AP, Woodward M, Royston P, Vergouwe Y, Altman DG, Grobbee DE. Risk prediction models: I. Development, internal validation, and assessing the incremental value of a new (bio)marker. Heart. 2012;98:683-90.

16. Murase H, Inokuchi M, Takagi Y, Kato K, Kojima K, Sugihara K. Prognostic significance of the co-overexpression of fibroblast growth factor receptors 1,2 and 4 in gastric cancer. Mol Clin Oncol. 2014;2:509-17.

17. Hase T, Kawashiri S, Tanaka A, Nozaki S, Noguchi N, Kato K, Nakaya H, Nakagawa K. Correlation of basic fibroblast growth factor expression with the invasion and the prognosis of oral squamous cell carcinoma. J Oral Pathol Med. 2006;35:136-9.

18. Dellacono FR, Spiro J, Eisma R, Kreutzer D. Expression of basic fibroblast growth factor and its receptors by head and neck squamous carcinoma tumor and vascular endothelial cells. Am J Surg. 1997;174:540-4.

19. Freier K, Schwaenen C, Sticht C, Flechtenmacher C, Mühling J, Hofele C, Radlwimmer B, Lichter P, Joos S. Recurrent FGFR1 amplification and high FGFR1 protein expression in oral squamous cell carcinoma (OSCC). Oral Oncol. 2007;43:60-6.

20. Streit S, Bange J, Fichtner A, Ihrler S, Issing W, Ullrich A. Involvement of the FGFR4 Arg388 allele in head and neck squamous cell carcinoma. Int J Cancer. 2004;111:213-7.

21. Ipenburg NA, Koole K, Liem KS, van Kempen PM, Koole R, van Diest PJ, van Es RJ, Willems SM. Fibroblast growth factor receptor family members as prognostic biomarkers in head and neck squamous cell carcinoma: a systematic review. Target Oncol. 2015. doi:10.1007/s11523-015-0374-9.

22. Dutra RL, de Carvalho MB, Dos Santos M, Mercante AM, Gazito D, de Cicco R, Group G, Tajara EH, Louro ID, da Silva AM. FGFR4 profile as a prognostic marker in squamous cell carcinoma of the mouth and oropharynx. PLoS One. 2012;7:e50747.

23. Bertz S, Abeé C, Schwarz-Furlan S, Alfer J, Hofstädter F, Stoehr R, Hartmann A, Gaumann AK. Increased angiogenesis and FGFR protein expression indicate a favourable prognosis in bladder cancer. Virchows Arch. 2014;465:687-95.

24. Sugiura K, Ozawa S, Kitagawa Y, Ueda M, Kitajima M. Coexpression of aFGF and FGFR-1 is predictive of a poor prognosis in patients with esophageal squamous cell carcinoma. Oncol Rep. 2007; 17:557-64.

25. Shin EY, Lee BH, Yang JH, Shin KS, Lee GK, Yun HY, Song YJ, Park SC, Kim EG. Up-regulation and co-expression of 
fibroblast growth factor receptors in human gastric cancer. J Cancer Res Clin Oncol. 2000;126:519-28.

26. Hagel M, Miduturu C, Sheets M, Rubin N, Weng W, Stransky N, Bifulco N, Kim JL, Hodous B, Brooijmans N, Shutes A, Winter C, et al. First selective small molecule inhibitor of FGFR4 for the treatment of hepatocellular carcinomas with an activated FGFR4 signaling pathway. Cancer Discov. 2015;5:424-37.

27. Packer LM, Pollock PM. Paralog-specific kinase inhibition of FGFR4: adding to the arsenal of anti-FGFR agents. Cancer Discov. 2015;5:355-7.

28. Nguyen PT, Tsunematsu T, Yanagisawa S, Kudo Y, Miyauchi M, Kamata N, Takata T. The FGFR1 inhibitor PD173074 induces mesenchymal-epithelial transition through the transcription factor AP-1. Br J Cancer. 2013;109:2248-58.

29. Seiwert TY, Zuo Z, Keck MK, Khattri A, Pedamallu CS, Stricker T, Brown C, Pugh TJ, Stojanov P, Cho J, Lawrence MS, Getz G, et al. Integrative and comparative genomic analysis of HPV- positive and HPV-negative head and neck squamous cell carcinomas. Clin Cancer Res. 2015;21:632-41.

30. Network Cancer Genome Atlas. Comprehensive genomic characterization of head and neck squamous cell carcinomas. Nature. 2015;517:576-82.

31. Cheng YM, Chou CY, Hsu YC, Chen MJ. Influence of HPV16 E6/7 on the expression of FGF2 and FGFR type B in cervical carcinogenesis. Reprod Sci. 2012;19:580-6.

32. Rosty C, Aubriot MH, Cappellen D, Bourdin J, Cartier I, Thiery JP, Sastre-Garau X, Radvanyi F. Clinical and biological characteristics of cervical neoplasias with FGFR3 mutation. Mol Cancer. 2005;4:15.

33. Göke F, Franzen A, Hinz TK, Marek LA, Yoon P, Sharma R, Bode M, von Maessenhausen A, Lankat-Buttgereit B, Göke A, Golletz C, Kirsten R, et al. FGFR1 expression levels predict BGJ398 sensitivity of FGFR1-dependent head and neck squamous cell cancers. Clin Cancer Res. 2015;21:4356-64. 